\title{
Fluorescence scanning near-field optical microscopy in reflection
}

\author{
Alain Jalocha, Marco H. P. Moers, Niek F. van Hulst \\ Applied Optics group, \\ Dept. of Applied Physics \& MESA Research Institute, \\ University of Twente, P.O. Box 217, 7500AE Enschede, the Netherlands
}

\begin{abstract}
A Fluorescence Scanning Near-Field Optical Microscope operated in reflection is presented. A pulled optical fiber is used both as an emitter for the exciting light and a collector for the generated fluorescence. The advantage of this set-up is the use of the fiber tip as an emitter and a collector. The sample is locally illuminated and no extra optical elements are needed for the detection. We will describe the shear force set-up which is used to control the tip to surface distance. Direct correlation between force map and optical signal is thus possible. Fluorescence images have been obtained on Langmuir-Blodgett films where we estimate the resolution at $200 \mathrm{~nm}$. Moreover the anisotropic property of the monolayer allows polarization contrast measurements. Thus, we show true optical contrast due to fluorescence and polarization is applicable to this configuration. Artifacts in LB films shear force image are discussed. Shear force approach curves obtained on glass and polymer domains are presented to explain the chemical origin of the phenomenon.
\end{abstract}

\section{INTRODUCTION}

Suggested in the beginning of the century [1], Scanning Near-field Optical Microscopy (SNOM) has been demonstrated in 1972 [2] with radiowave frequency. The technique is analogous to Scanning Tunneling Microscopy (STM) and Atomic Force Microscopy (AFM) but at variance the optical interaction is studied. The probe size and its separation with the sample determine the image resolution. In recent years several schemes have been developed with different probes and illumination methods [3]. The shear force detection [4,5] combined with the use of pulled fiber allows to control the working distance and to avoid tip and/or sample damage. It gives simultaneously a force image of the sample. Fluorescence SNOM in transmission has been demonstrated in 1986 with a resolution of $50 \mathrm{~nm}$ [6]. In this case a true optical contrast coming from the sample property is displayed. This is of a great interest in detection and characterization of single molecules [7,8]. This technique is promising in biology too: lipid films [9] and labeled chromosomes [10] investigations have been reported with $100 \mathrm{~nm}$ resolution.

We present fluorescence images of Langmuir-Blodgett films obtained with an equivalent system as proposed by Spajer et al [11,14]. Analyzing the fluorescence intensity we determine the resolution at $200 \mathrm{~nm}$. We show that polarization contrast (already reported in classical microscopy [15] and SNOM in transmission [9]) is a technique which can be applied in our system. This results are promising and obviously helpful in solid states physics and studies of non transparent samples.

Although it is useful in SNOM the shear force mechanism is not totally explained. We present results showing specific chemical origin of the phenomenon. 


\section{MICROSCOPIC SET-UP}

The near field probe is an adiabatically pulled fiber. The extremity of a multimode fiber is pulled with a commercial instrument (Sutter P2000) and its diameter is reduced to about $100 \mathrm{~nm}$. In the present works the probe is not coated with metal, and used both as an emitter for the exciting wavelength and a collector for the fluorescence. We choose a multimode fiber to collect the maximum fluorescence signal.

The sample to probe distance regulation is the central part of any near field microscope. Although a tunneling feedback [16] has been developed, the shear force technique is the most popular distance control in SNOM using pulled fiber. The principle has been presented three years ago $[4,5]$. The fiber is mounted on a piezo-ceramic and is vibrated at the first resonance frequency. Close to the surface forces act on the tip and reduce the dither vibration. Using a feedback system a z-piezo controls the tip to sample distance. Several schemes have been proposed to detect the vibration amplitude [17,18]. This is done in our system by using an external optical method (figure 1).

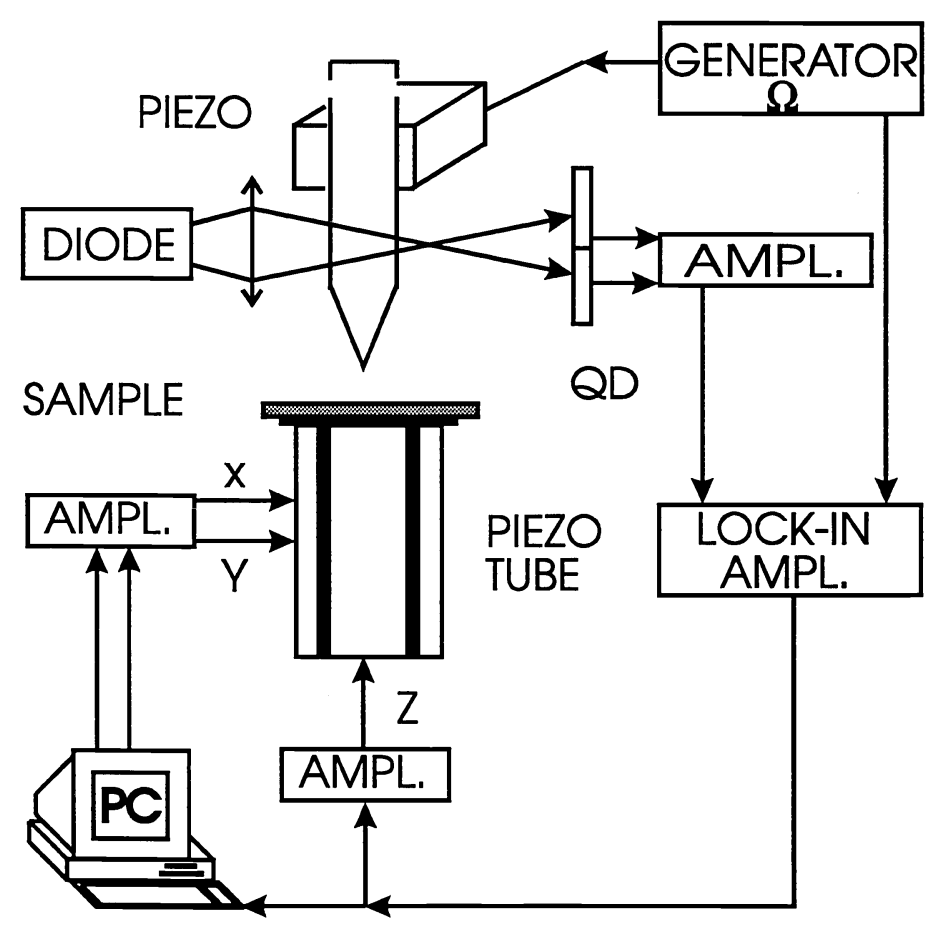

Figure 1: The shear-force set-up

The shear force detection system is easily implemented and the detection is independent of the sample properties. Perpendicular to the fiber a laser diode is focused in front of the extremity and a split detector collects the diffracted light.

Figure 2 shows an example of the shear force signal with a vibration amplitude of $80 \mathrm{~nm}$. The sample is an $\mathrm{Al}$ mirror. One notes the behaviour of the approach curve changes within the last $12 \mathrm{~nm}$. This value can be reduced using a smaller amplitude [17]. During the scan the probe follows the surface contours and the voltage needed to keep the shear force signal constant displays a force map. 


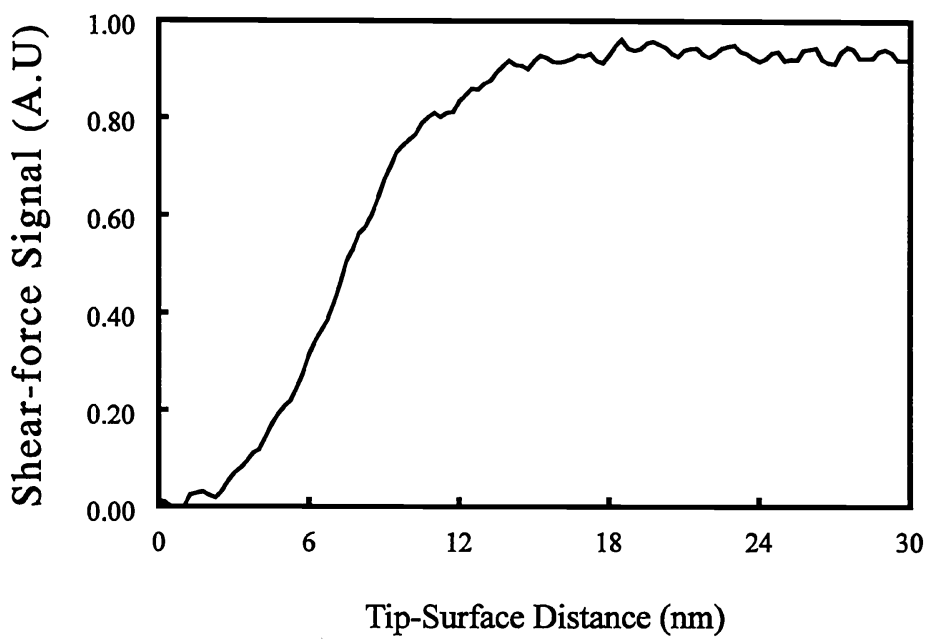

Figure 2: Shear force approach curve with uncoated fiber towards an Al surface.

The optical arrangement is shown figure 3. An Ar-ion laser $(\lambda=514$ $\mathrm{nm})$ is launched into the fiber. A dichroic mirror (DM 580 Nikon, $\lambda$ $=560 \mathrm{~nm})$ and a high pass filter (BA 590 Nikon, $\lambda>590 \mathrm{~nm}$ ) are used to separate the excitation and fluorescence beams coming from the sample. A polarizes and an analyzer control the input and output polarization. A photomultiplier tube (Hamamatsu R5600) collects the fluorescence signal.

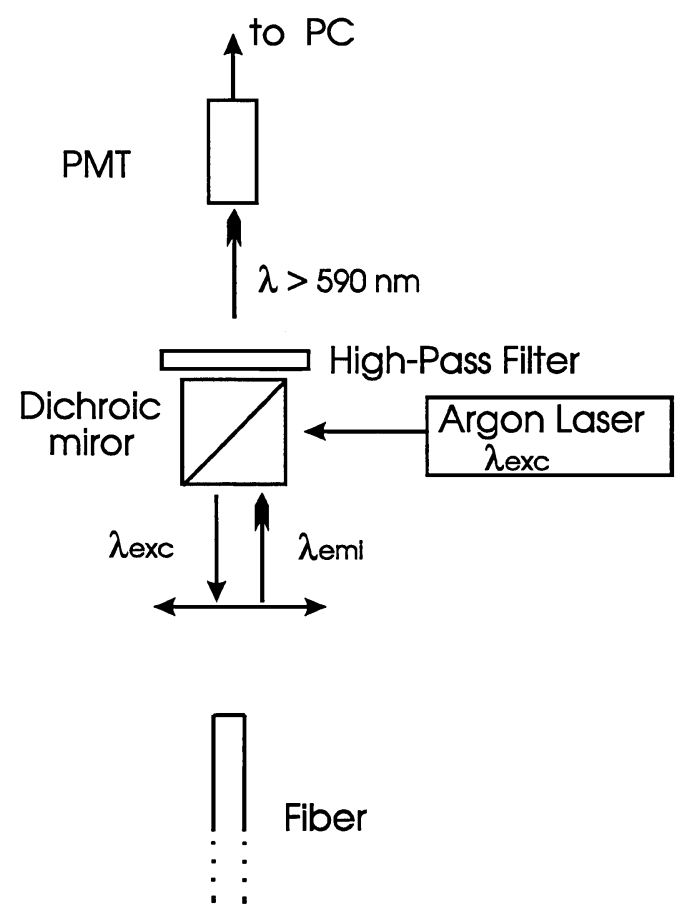

Figure 3: Optical arrangement for fluorescence detection.

A piezo-tube allows the sample displacement in the $\mathrm{x}$ and $\mathrm{y}$ directions with a maximum scale of 20 $\mu \mathrm{m}^{2}$. A personal computer generates the scan pattern and acquires the force and fluorescence signals. The feedback system limits the data acquisition at $300 \mathrm{~Hz}$. 


\section{RESULTS AND DISCUSSION}

\subsection{Sample description}

Ultrathin lipid films are ideal test structures for near-field optical microscopy. We have investigated a Diethylene glycol Diamine PentacosaDiynoic Amide (DPDA) monolayer polymerized by Ultra-Violet. Prepared by LB technique [15] a monolayer with a thickness of $6 \mathrm{~nm}$ is obtained on a glass slide. It is composed of domains. Although they show a strong absorption in the green and emission in the red, each domain has a particular geometry (several square micrometers in size) and a distinct crystalline orientation. Thus each domain is characterized by its own absorption and emission moments.

\subsection{Fluorescence measurements}

Topographic and fluorescence maps are shown in figure 4 . The scan area is $12 * 12 \mu \mathrm{m}^{2}$ with $200 * 200$ pixels. We note on the force image (a) that the film is composed of several domains. Although its thickness is $6 \mathrm{~nm}$ it seems to be lower than the glass parts. This behavior will be clarified in the next section. In the fluorescence map (figure $4 \mathrm{~b}$ ) obtained without polarizes and analyzer, we clearly recognize domains. The parts where no film covers the glass appear black.

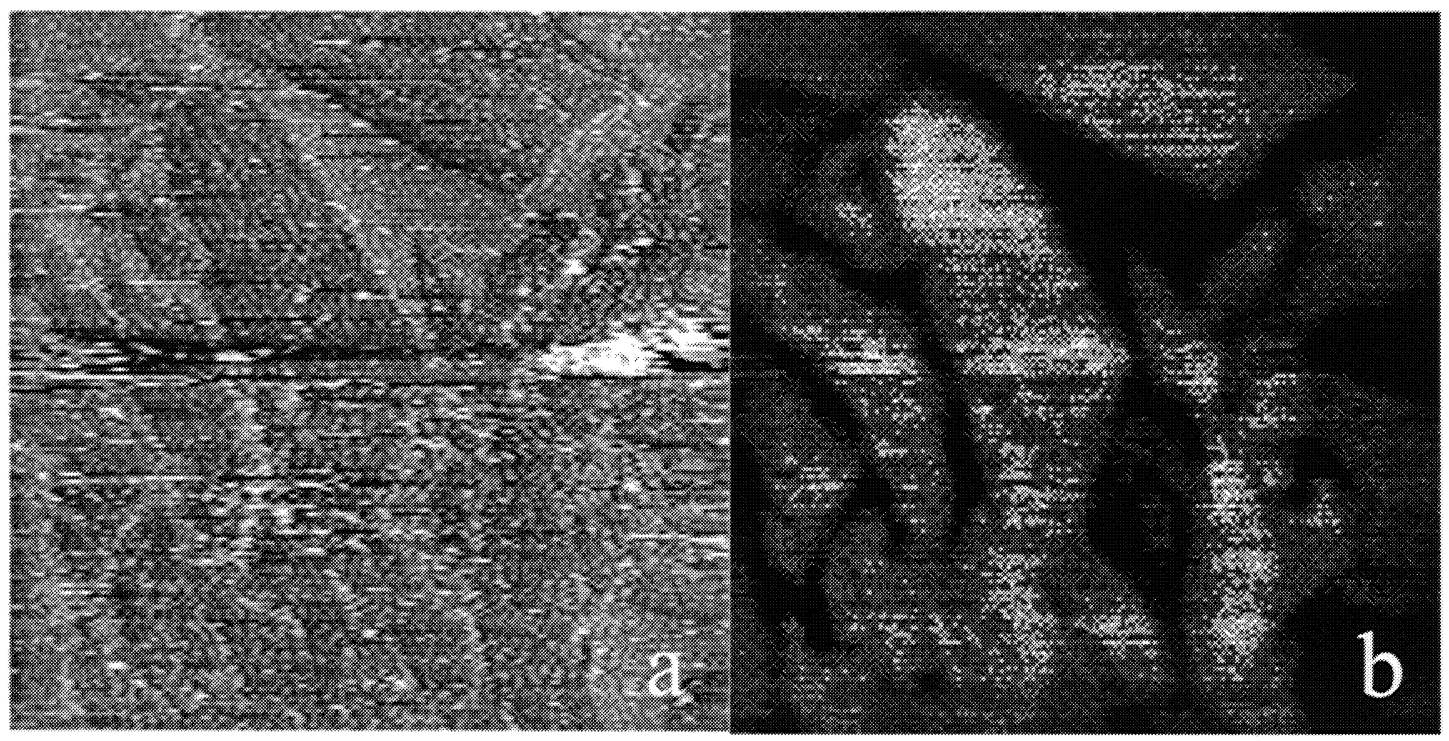

Figure 4: Fluorescence image of DPDA film. (a) is the shear force image and (b) the corresponding fluorescence map.

In figure 5 a fluorescence contour line is plotted. We define the resolution as the width between Maximum $-20 \%$ and Minimum $+20 \%$. We estimate the resolution at $200 \mathrm{~nm}$. 


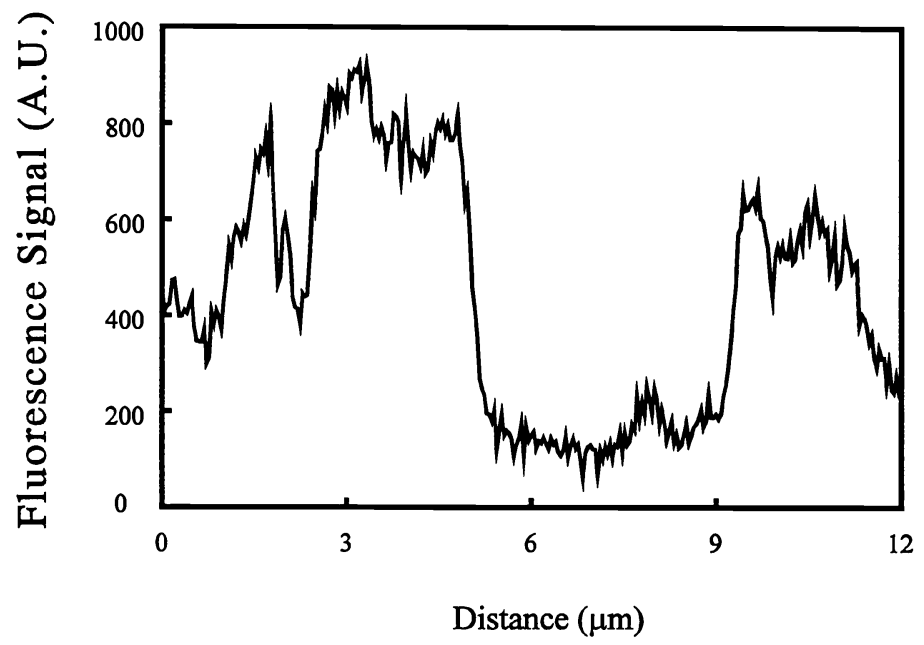

Figure 5: Contour line of the fluorescence image $4 b$

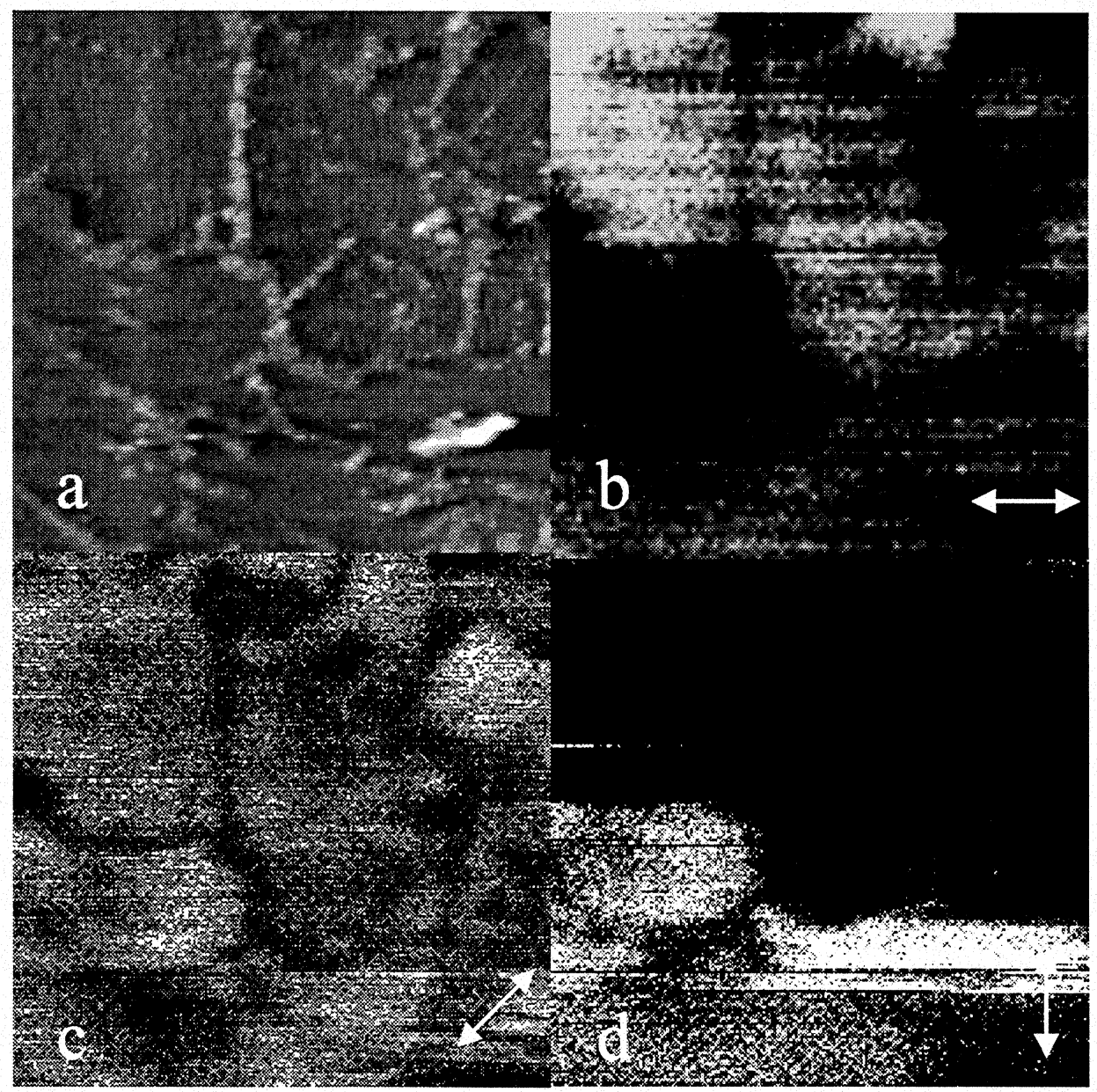

Figure 6: Shear force (a) and fluorescence images of DPDA film.

The input polarization is visualized by the arrows. 
Figure 6 shows a scan of another part of the DPDA film. The scan area is $10,4 * 10,4 \mu \mathrm{m}^{2}$ with $200 * 200$ pixels. Figure $6 a$ is the shear force image. Figures $6 b, 6 c, 6 d$ are fluorescence images for different input linear polarization directions with an orientation of $0^{\circ}, 45^{\circ}$ and $90^{\circ}$, respectively.

We measured the maximum fluorescence level at $1,3 \mathrm{pW}$ for a $1 \mu \mathrm{W}$ output of the tip. We note the evolution of the relative fluorescence intensities of the different domains with the change of polarization. The fluorescence intensity depends on absorption and emission moments too, and its level is maximum when the input polarization is parallel to the absorption moment. One can deduce from figures $6 \mathrm{~b}$ and $6 \mathrm{~d}$ that the upper domains of the studied site have the same characteristics. The same conclusions can be drawn for the lower part of the image. Domains with perpendicular orientations appear with inverted level between $6 \mathrm{~b}$ and $6 \mathrm{~d}$. Figure $6 \mathrm{c}$ corroborates these remarks. In this image all domains fluoresce because they are partially excited with an input polarization of $45^{\circ}$.

\subsection{The shear force contrast mechanism}

In order to test the shear force on thin film, we imaged an aluminum layer with traces of latex spheres. It has been fabricated by an aluminum deposition on a spheres monolayer which has been removed by ultrasound. The hole diameter is $481 \mathrm{~nm}$. Figure 7 is the shear force image of a part of this sample. The scan area is $4 * 4 \mu \mathrm{m}^{2}$ and the pixel size is $20 \mathrm{~nm}$. In this case the force image represents the "real" topographic map of the sample. The height of the aluminum layer has been measured to $12 \mathrm{~nm}$ by both shear force and conventional atomic force technique. It is clear that forces acting on the tip are merely dependent on the topography. We can clearly recognize the circular holes in the film. The geometry and the compacity of the spheres give rise to clusters whose are well resolved especially in the upper left corner of the image.

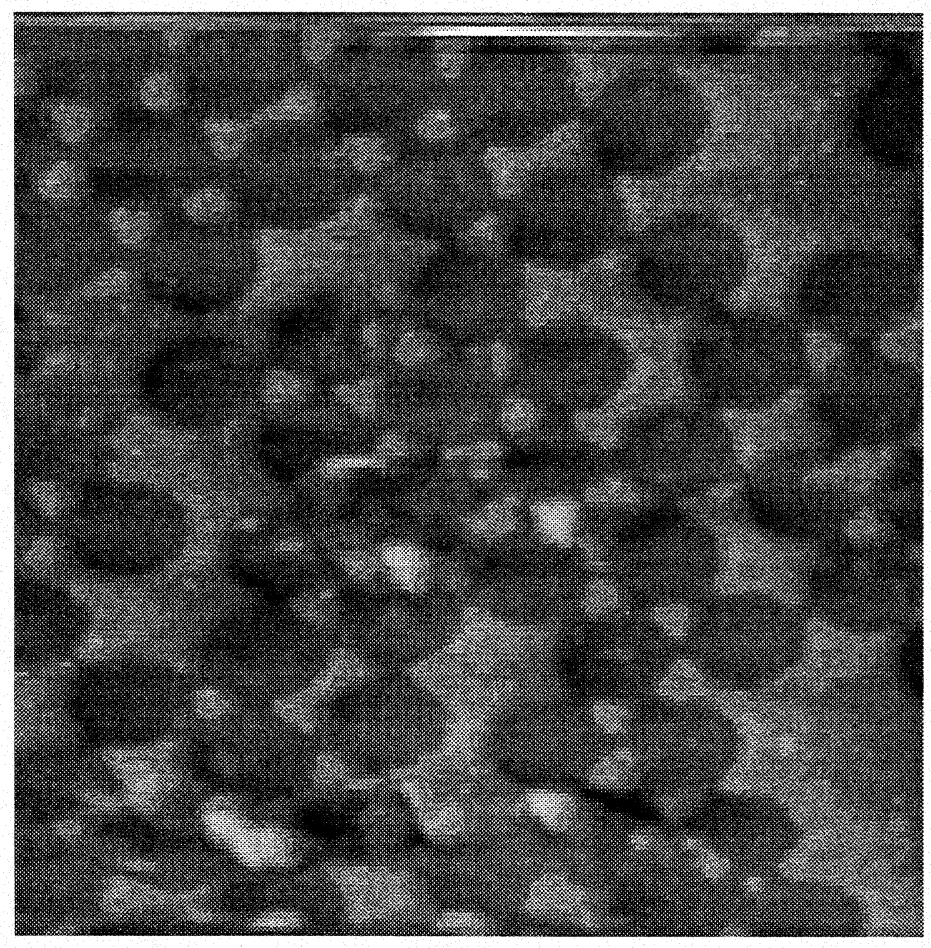

Figure 7: Shear force image of latex spheres traces in an aluminum layer 
As we mentioned in the last section, the darker areas in shear force images of Langmuir-Blodgett films show strong fluorescence signal which means those areas are film domains. In this case the shear force map is no more a topographic map.

Figure 8 shows two shear force approach curves. (a) is obtained on DPDA film and (b) and parts where no film covers the glass.

One note the slopes of these two graphs are different. The shear force damping mechanism is weaker on polymer than on the glass. Choosing a set-point, the tip has to be closer to the polymer surface to keep an equivalent shear force signal despite the $6 \mathrm{~nm}$ topographic height of the polymer. An explanation of this phenomenon can be the chemical nature of the sample. The same sample has been studied by adhesion mode atomic force microscopy [19]. The conclusion of this study is that the film is less hydrophilic than glass. The contrast in adhesion force image is thus inverted with respect of the topography. These two results are consistent and we conclude the shear force mechanism is depending on adhesion force between the tip and the sample. Thus the probe does not follow the contours of the sample with a constant distance, and gives rise to this artifact. This aspect of the shear force mechanism will be subject of further research.

\section{Shear-Force Dependence L.B Films Sample}

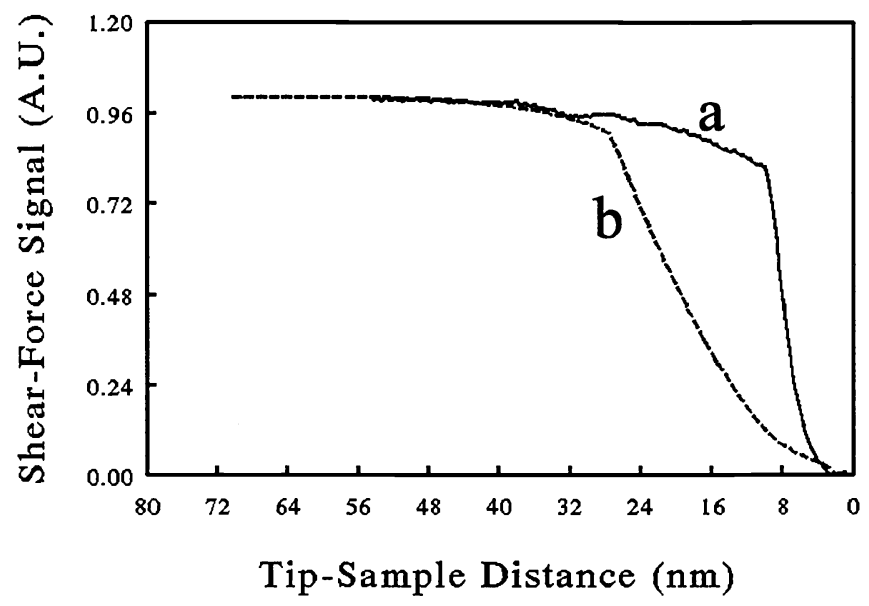

Figure 8: Shear force approach curves on (a) DPDA film and (b) glass

\section{SUMMARY}

We presented fluorescence measurements with polarization contrast using a Scanning Near-field Optical Microscope in reflection. A tapered non metallized optical fiber is used to collect the fluorescence signal it created itself. Fluorescence and non fluorescence areas can be distinguished with $200 \mathrm{~nm}$ resolution. Moreover we demonstrated polarization contrast on LB films which allows to characterize the orientation of polymer backbone. These results are promising because they show that contrast mechanism useful in classical microscopy and SNOM in transmission can be applied in the reflection set-up. It opens new possibilities in solid states physics and in the study of non transparent samples. We showed chemical dependence of shear force approach curves on LB films. Thus the shear force images on DPDA film show its hydrophobicity and not its topography. Although this behavior is an artifact of the method it can be used to characterize the sample chemical property. 


\section{ACKNOWLEDGMENTS}

The Langmuir-Blodgett films samples have been prepared by Uli Hoffmann and Hermann E. Gaub of the Technical University of Münich. This work was supported by the European Commission for the Research (contract: ERBCHGCT 920049). Alain Jalocha thanks the faculty of Twente, the MESA Institute and the staff of the TOP laboratory.

\section{REFERENCES}

1. E. A. Synge, Phil. Mag. 6, 356-358 (1928).

2. $\quad$ E. A. Ash, G. Nicholls, "Super-resolution Aperture Scann. Micr.", Nature 237, 510-512 (1972).

3. D. Pohl, in Advances in Optical and electron microscopy, Acad. Press, New York, pp. 243-312 (1991)

4. E. Betzig, P. L. Finn, J. S. Weiner, "Combined Shear Force and Near-Field Scanning Optical Microscopy", Appl. Phys. Lett. 60, 2484-2486 (1992)

5. R. Toledo-Crow, P. C. Yang, Y. Chen, M. Vaez-Iravani, Near-field differential scanning optical microscope with atomic force regulation", Appl. Phys. Lett. 60, 2957-2959 (1992)

6. E. Betzig, A. Lewis, A. Harootunian, M. Isaacson, E. Kratschmer, "Near-Field Scanning Optical Microscopy", Biophys. J. 49, 269-279 (1986)

7. E. Betzig, R. Chichester, "Single Molecules Observed by Near-Field Scanning Optical Microscopy", Science 262, 1422-1424 (1993)

8. R. Kopelman, W. Tan, "Near-Field Opt.: Imaging Single Molecules", Science 262, 1382-1384 (1993)

9. M. H. P. Moers, H. E. Gaub, N. F. van Hulst, "Polydiacetylene Monolayers studied with a Fluorescence Scanning Near-Field Optical Microscope", Langmuir 10, 2774-2777 (1994)

10. N. F. van Hulst, M. H. P. Moers, E. Borgonjon, "Applications of Near Field Optical Microscopy", proceeding workshop "Photon \& Local Probes", Reichenau 12-16 Sept. 1994, to be published in NATO ASI series E, Kluwer Acad. Publ. (1995).

11. M. Spajer, A. Jalocha, "The reflection near field optical microscope an alternative to STOM", Near Field Optics, Ed. D. W. Pohl and C. Courjon, Kluwer Acad., pp. 87-94 (1993)

12. A. Jalocha, C. Pieralli, "A scanning optical profilometer using the SNOM architecture", Pure Appl. Opt. 3, 793-804 (1994)

13. A. Jalocha, N. F. van Hulst, "Dielectric and Fluorescent Samples imaged by Scanning Near-Field Optical Microscopy in Reflection", to be published in Opt. Comm. (1995).

14. A. Jalocha, N. F. van Hulst, "Polarissation contrast in Near field optical microscopy in reflection", to be published in J. Opt. Soc. Am. B. (1995).

15. B. M. Goettgens, R. W. Tillmann, M. Radmacher, H. E. Gaub, "Molecular Order in Polymerizable Langmuir-Blodgett Films by Microfluorescence and Scanning Force Microscopy", Langmuir 8, 17681774 (1992).

16. M. Garcia-Parajo, E. Cambril, Y. Chen, "Simultaneous scanning tunneling microscope and collection mode scanning near-field optical microscope using coated optical fibreprobes", Appl. Phys. Lett. 65, 1498-1500 (1994)

17. M. H. P. Moers, N. F. van Hulst, A. G. T. Ruiter, B. Bölger, "Optical contrast in near-field techniques", Ultramicroscopy 57, 298-302 (1995)

18. J-K. Leong, C. C. Williams, "Shear force microscopy with capacitance detection for near-field scanning optical microscopy", Appl. Phys. Lett. 66, 1432-1434 (1995)

19. K. O. van der Werf, C. A. J. Putman, B. G. de Grooth, J. Greve, "Adhesion force imaging in air and liquid by adhesion mode atomic force microscopy”, Appl. Phys. Lett. 65, 1195-1197 (1994) 